\title{
Collinearity isn't a disease that needs curing
}

\author{
Jan Vanhove \\ University of Fribourg
}

\begin{abstract}
Once they have learnt about the effects of collinearity on the output of multiple regression models, researchers may unduly worry about these and resort to (sometimes dubious) modelling techniques to mitigate them. I argue that, to the extent that problems occur in the presence of collinearity, they are not caused by it but rather by common mental shortcuts that researchers take when interpreting statistical models and that can also lead them astray in the absence of collinearity. Moreover, I illustrate that common strategies for dealing with collinearity only sidestep the perceived problem by biasing parameter estimates, reformulating the model in such a way that it maps onto different research questions, or both. I conclude that collinearity in itself is not a problem and that researchers should be aware of what their approaches for addressing it actually achieve.
\end{abstract}

Keywords: regression assumptions, multiple regression, interpreting regression models

As researchers and students learn more about statistical models, they sooner or later stumble across the term (multi)collinearity. Collinearity, which roughly means that the predictors in a statistical model are correlated with each other, is often cast as a problem for statistical analysis. This suggests that the conscientious analyst has to solve it. I will argue that, to the extent that problems occur in the presence of collinearity, these are not caused by the collinearity itself but rather by a faulty way of thinking about statistical models that can lead analysts astray even in the absence of collinearity. Common strategies for dealing with collinear predictors do not solve these perceived problems but instead sidestep them, often by fitting a model that, perhaps unbeknownst to the analyst, answers a different set of questions from the original one.

This article does not present any novel insights, but I hope that it will nonetheless be educational to readers who sometimes find the output of regression models befuddling. I will focus on collinearity between two continuous predictors in (ordinary least squares) multiple regression models. In this case the strength of the collinearity can be gauged from the correlation between the predictors. However, all of my points apply to models with categorical predictors or a mix of categorical and continuous predictors as well. I will not discuss methods for assessing the degree of collinearity between three or more predictors for the simple reason that I find them a distraction: in what follows, I will argue that collinearity is not a statistical problem and should not be checked for (also see O'Brien, 2007).

\section{Collinearity and its consequences}

Collinearity means that a substantial amount of information contained in some of the predictors included in a statistical model can be pieced together as a linear combination of some of the other predictors in the model. The easiest case is when you have a multiple linear regression model with two correlated predictors, as in the examples to follow. These predictors can be continuous or categorical, but I will stick to continuous predictors for ease of exposition.

I created four datasets with two continuous predictors to illustrate collinearity and its consequences. You can find the $\mathrm{R}$ code to reproduce all analyses at https: //osf.io/jupd8/. The outcome in each dataset was created using the following equation; the parameter values 
were chosen arbitrarily:

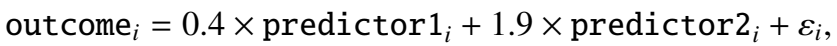

where the residuals $\left(\varepsilon_{i}\right)$ were drawn from a normal distribution with a standard deviation of $3.5 .^{1}$

The four datasets are presented in Figures 1 through 4. In Figure 1, a linear function of predictor 1 captures most of the information contained in predictor2, so the two predictors are strongly collinear. In Figure 3, by contrast, both predictors are completely unrelated, and a linear function of one predictor cannot capture any information in the other. Hence, the two predictors are not collinear at all. Some readers may be surprised to see that I consider a situation where two predictors are correlated at $r=0.50$ (Figure 2) to be a case of weak rather than moderate or strong collinearity. But in fact, the consequences of having two predictors that are correlated at $r=0.50$ (rather than at $r=0.00$ ) are negligible. Finally, Figure 4 highlights the linear part in collinearity: while the two predictors in this figure are related in that predictor 2 perfectly determines predictor 1 , there is no linear relationship between them whatsoever. (You cannot uniquely determine the value for predictor 2 when you know the value for predictor1, though.) The dataset in Figure 4 is not affected by any of the statistical consequences of collinearity, but it will be useful to illustrate a point I want to make below.

To illustrate the statistical consequences of collinearity, I simulated 10,000 samples of 50 observations in which the two predictors were highly correlated (sample correlation of $r=0.98$, yielding datasets similar to the one in Figure 1) and 10,000 samples of 50 observations in which they were completely orthogonal (sample $r=0.00$, yielding datasets similar to the one in Figure 3 ). In all cases, both predictors were independently related to the outcome according to Equation 1. On each simulated sample, I ran a multiple regression model from which I extracted the estimated model coefficients. Figure 5 shows the estimated coefficients for the first predictor, whose true parameter value is 0.4 . Clearly, the estimates vary more when the predictors are strongly correlated than when they are not, such that individual estimates can lie farther from the true parameter value and often have the opposite sign from this true parameter value. However, on average, the estimates equal the true parameter value. In statistics parlance, they are "unbiased."

Crucially, and happily, this greater variability is reflected in the standard errors and confidence intervals around these estimates: The standard errors and confidence intervals are automatically wider when the estimated coefficients are affected by collinearity. This is
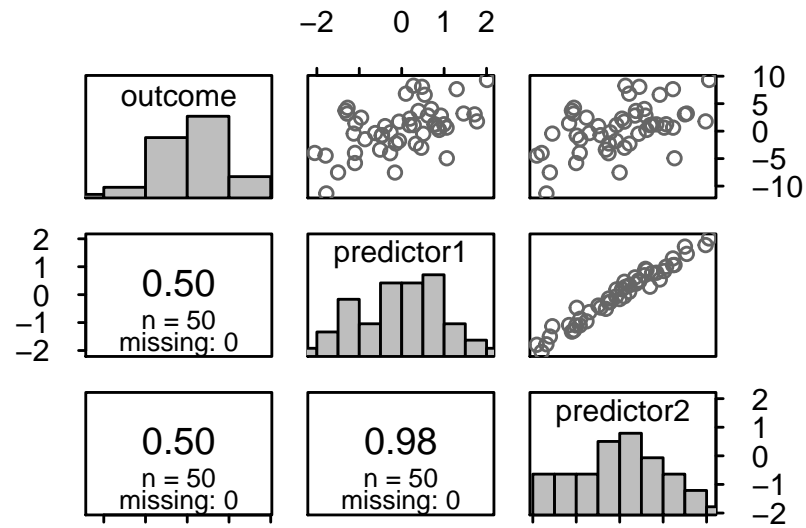

$\begin{array}{lll}-10 & 0 & 10\end{array}$

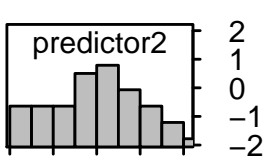

Figure 1. A dataset with strongly collinear predictors $(r$ $=0.98)$.

illustrated in Figure 6: If you fit multiple regressions on the datasets plotted in Figures 1 to 4, the confidence intervals are considerably wider if the predictors are strongly collinear than when they are not. Moreover, the confidence intervals retain their nominal coverage rates (i.e., $x \%$ of the $x \%$ confidence intervals contain the true parameter value). So the statistical consequence of collinearity is automatically taken care of in the model's output and requires no additional computations on the part of the analyst.

The greater variability in the estimates, and the appropriately larger standard errors and wider confidence intervals all reflect a relative lack of information in the sample (also see Morrissey and Ruxton, 2018). It is difficult to improve on York's (2012) explanation of the problem and possible solutions:

"Collinearity is at base a problem about information. If two factors are highly correlated, researchers do not have ready access

\footnotetext{
${ }^{1}$ Some researchers examining the consequences of collinearity generate both the predictors and the outcome directly from multivariate normal distributions in which the correlation between the predictors varies but the correlations between the outcome and the individual predictors do not (e.g., Wurm and Fisicaro, 2014) rather than generating the outcome as a function of the predictors as I did. In doing so, they implicitly allow the true regression equation to vary from simulation to simulation: If you fix the correlations between the predictors and the outcome, but you want to vary the intercorrelation between the predictors, you have to vary the $\beta$ parameters (fixed at 0.4 and 1.9 in my example) and the $\sigma$ parameter (fixed at 3.5 in my example). The result of this is that such simulations may paradoxically show that you obtain more significant estimates when the predictors are strongly collinear (see Wurm and Fisicaro, 2014, Table 6), but they actually compare different data generating processes.
} 


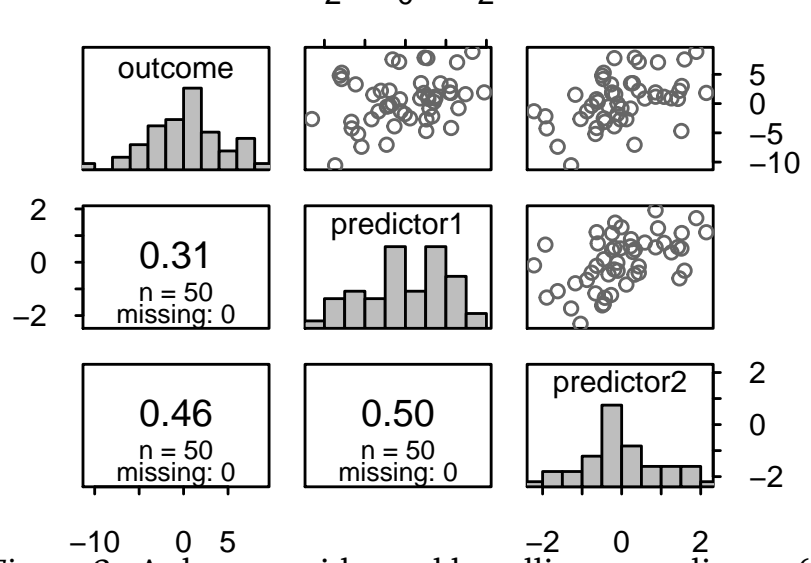

Figure 2. A dataset with weakly collinear predictors $(r$ $=0.50)$.

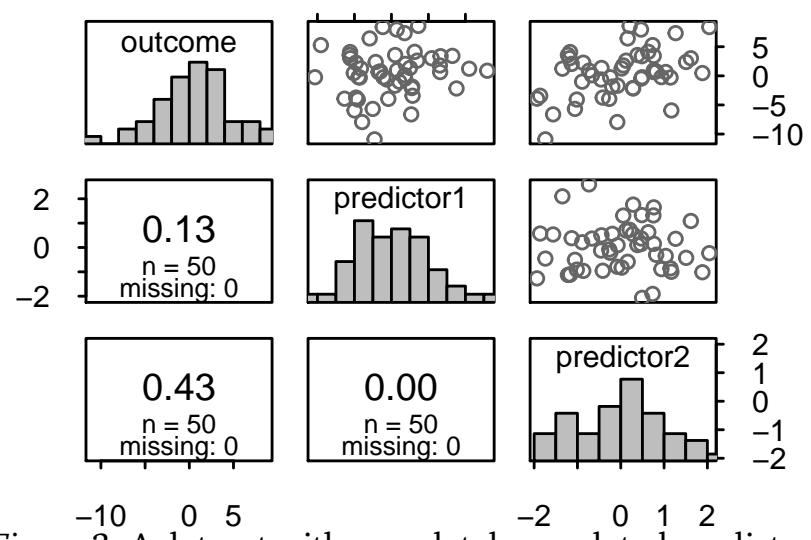

Figure 3. A dataset with completely unrelated predictors $(r=0.00)$.

$$
\begin{array}{lll}
-1.5 & 0.0 & 1.5
\end{array}
$$
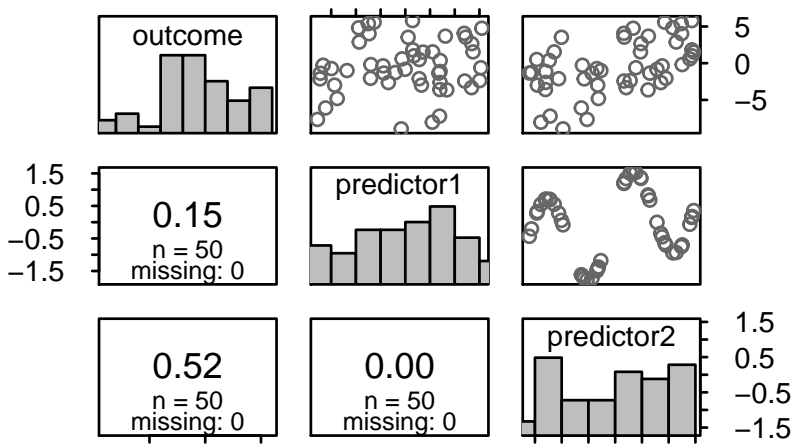

$\begin{array}{lll}-5 & 0 & 5\end{array}$

$\begin{array}{lll}-1.5 & 0.0 & 1.5\end{array}$

Figure 4. A dataset with orthogonal $(r=0.00)$ but perfectly related predictors: Once you know the value of predictor2, you know the value of predictor 1 . to much information about conditions of the dependent variables when only one of the factors actually varies and the other does not. If we are faced with this problem, there are really only three fundamental solutions: (1) find or create (e.g. via an experimental design) circumstances where there is reduced collinearity; (2) get more data (i.e. increase the $\mathrm{N}$ size), so that there is a greater quantity of information about rare instances where there is some divergence between the collinear variables; or (3) add a variable or variables to the model, with some degree of independence from the other independent variables, that explain(s) more of the variance of $\mathrm{Y}$, so that there is more information about that which is being modeled." (p. 1384)

\section{Is collinearity a problem?}

For the most part, I think that collinearity is a problem for statistical analyses in the same way that Belgium's lack of mountains is detrimental to the country's chances of hosting the Winter Olympics: It is an unfortunate fact of life, but not something that has to be solved. The three solutions that York (2012) mentions, i.e., running another study, obtaining more data or reducing the error variance using covariates, are all sensible, but if you have to work with the data that you have, the model output will be unbiased and will appropriately reflect the degree of uncertainty in the estimates.

So I do not consider collinearity a problem. What is the case, however, is that collinearity highlights problems with the way many people think about statistical models and inferential statistics. Let's look at a couple of these.

\section{"Collinearity decreases statistical power."}

You may have heard that collinearity decreases statistical power, i.e., the chances of obtaining a statistically significant coefficient estimate if the true parameter value is different from zero. This is true, but the lower statistical power is a direct result of the larger standard errors, which appropriately reflect the greater sampling variability of the estimates. This is only a problem if you interpret "lack of statistical significance" as "zero effect." But then the problem does not lie with collinearity but with the belief that non-significant estimates indicate zero effects. (Schmidt (1996) calls this false belief "the most devastating of all to the research enterprise" (p. 126).) It is just that this false belief is even more likely than usual to lead you astray 


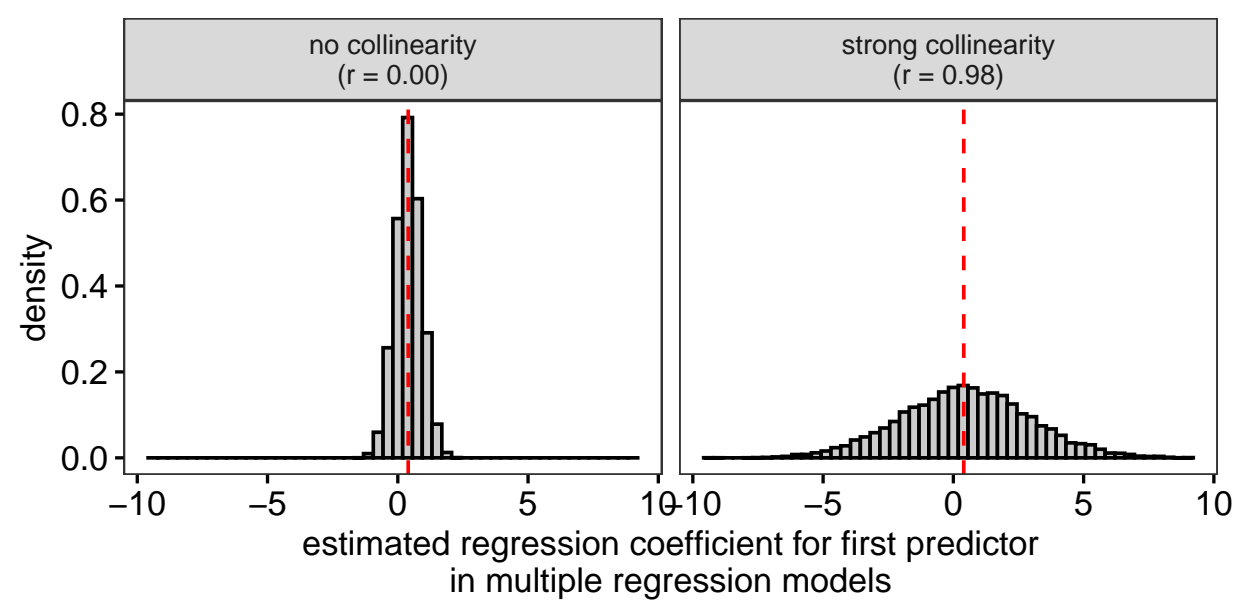

Figure 5. The parameters for the first predictor in 10,000 samples as estimated by multiple linear regression models. When the predictors are strongly collinear, the estimates vary more from sample to sample, but the estimates are unbiased in either case. The dashed vertical lines show the true parameter value (0.4).

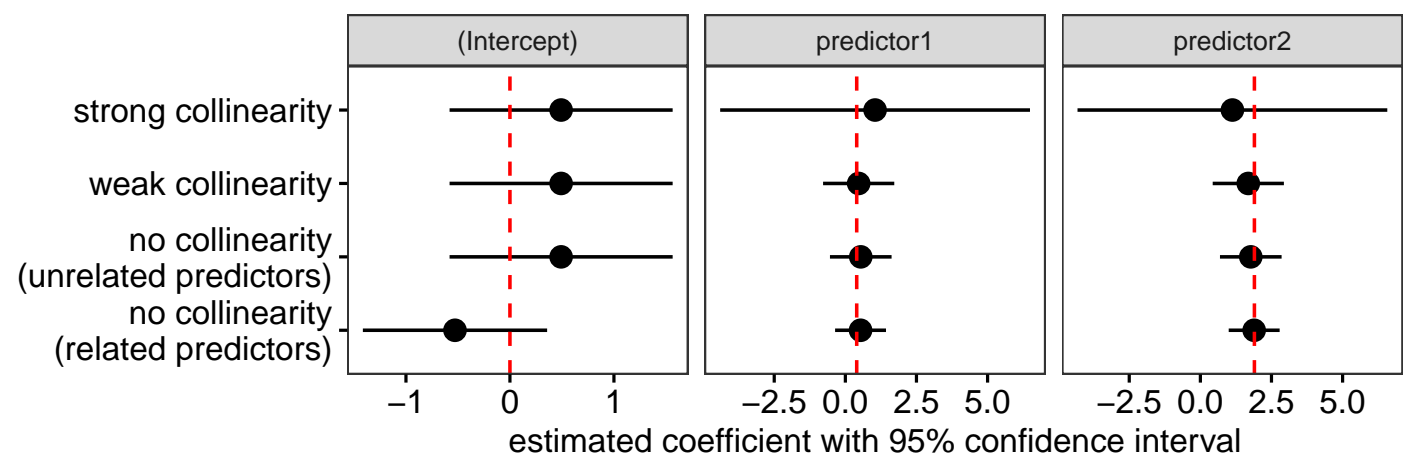

Figure 6. Estimated coefficients and their 95\% confidence intervals for the models fitted to the four datasets. The dashed vertical lines show the true parameter values.

when your predictors are collinear. If instead of focusing solely on the $p$-value, you take into account both the estimate and its uncertainty interval, then there is no problem.

Incidentally, I think that some people may be misled when they hear that collinearity "decreases" statistical power or "increases" standard errors as this wording may be taken to suggest that collinearity is a process that can be halted or reversed. It is true that compared to situations in which there is less or no collinearity and all other things are equal, the standard errors are larger and statistical power is lower when there is stronger collinearity. But outside of computer simulations, you cannot reduce collinearity while keeping all other things equal. In the real world, collinearity is not an unfolding process that can be nipped in the bud without bringing about other changes in the research design, the sampling procedure, or the statistical model and its interpretation.

Similarly, you may have heard that collinearity "inflates" standard errors or $p$-values. This wording, too, is misleading as it suggests that, in the presence of collinearity, standard errors and $p$-values are larger than they should have been. They are not, as per the discussion in the previous section (see Morrissey and Ruxton, 2018).

"None of the predictors is significant but the overall model fit is."

With collinear predictors, you may end up with a statistical model for which the $F$-test of the overall model fit is highly significant but that does not contain a single significant predictor. This is illustrated in Table 1 . The overall model fit for the dataset with strong collinearity (see Figure 1) is highly significant, but as shown in Figure 6, neither predictor has an estimated coefficient that is significantly different from zero: Both $95 \%$ confidence intervals contain zero.

If this seems strange, you need to keep in mind that the tests for the individual coefficient estimates and the test for the overall model fit seek to answer different 
Table 1

F-tests and p-values for the overall model fit for the multiple regression models on the four datasets. Even though neither predictor has a significant estimated coefficient in the 'strong collinearity' dataset (as shown in Figure 6), the overall fit is highly significant.

\begin{tabular}{llr} 
Dataset & F-test & p-value \\
\hline strong collinearity & $\mathrm{F}(3,47)=8.0$ & 0.001 \\
weak collinearity & $\mathrm{F}(3,47)=6.6$ & 0.003 \\
no collinearity (unrelated predictors) & $\mathrm{F}(3,47)=5.9$ & 0.005 \\
no collinearity (related predictors) & $\mathrm{F}(3,47)=9.8$ & 0.000 \\
\hline
\end{tabular}

questions, so there is no contradiction if they yield different answers. To elaborate, the test for the overall model fit asks if all predictors jointly can account for variance in the outcome; the tests for the individual coefficients ask whether these are different from zero. With collinear predictors, it is possible that the answer to the first question is "yes" and the answer to the second is "I have no idea." The reason for this is that with collinear predictors, either predictor could act as the stand-in of the other so that, as far as the model is concerned, either coefficient could well be zero, as long as the other is not. But due to the lack of information in the collinear sample, it is not sure which, if any, is zero (see McElreath, 2020, Chapter 6, for a lucid explanation).

So again, there is no real problem: The tests answer different questions, so they may yield different answers. It is just that when you have collinear predictors, this tends to happen more often than when you do not.

\section{"Collinearity means that you can't take model coef- ficients at face value."}

It is sometimes said that collinearity makes it more difficult to interpret estimated model coefficients. But the appropriate interpretation of an estimated regression coefficient is always the same, regardless of the degree of collinearity: According to the model, what would the difference in the mean outcome be if you took two large groups of observations that differed by one unit in the focal predictor but whose other predictor values were the same. The emphasised clause is crucial, and note the absence of any appeal to causality in the previous sentence. The interpretational difficulties that become obvious when there is collinearity are not caused by the collinearity itself but by mental shortcuts that people take when interpreting regression models.

For instance, you may obtain a coefficient estimate in a multiple regression model with collinear predictors that you interpret to mean that older children perform more poorly on a foreign-language (L2) writing task than younger children. This would be counterintuitive, and you may find that, in your sample, older children actually outperform younger ones. You could chalk this one up to collinearity, but the problem really is related to a faulty mental shortcut you took when interpreting your model: You forgot to take into account the crucial "but whose other predictor values are the same" clause. If your model also includes measures of the children's previous exposure to the L2, their motivation to learn the L2, and their L2 vocabulary knowledge, then what the estimated coefficient means is emphatically not that, according to the model, older children perform on average more poorly on a writing task than younger children. What it means is that, according to the model, older children perform more poorly than younger children with the same values on the previous exposure, motivation, and vocabulary knowledge measures. If, on reflection, this is not what you are actually interested in, then you should fit a different model (also see Miller and Chapman, 2001, for a similar point in the context of analysis of covariance). For instance, if you are interested in the overall difference between younger and older children regardless of their previous exposure, motivation and vocabulary knowledge, do not include these variables as predictors. But then you should have also not included these predictors if the collinearity had not been as strong.

Another interpretational difficulty emerges if you recast the interpretation of the estimate as follows: According to the model, what would the expected difference in mean outcome be if you took an observation and increased its value on the focal predictor by one unit but kept the other predictor values constant? The difference between this interpretation and the one that I offered earlier is that we have moved from a purely descriptive one to both a causal and an interventionist one (viz., the idea that one could change some predictor values while keeping the others constant and that this would have an effect on the outcome). In the face of strong collinearity, it becomes clear that this interventionist interpretation may be wishful thinking: It may be impossible to change values in one predictor without also changing values in the predictors that are collinear with it. But the problem here again is not the collinearity but the mental shortcut in the interpretation. Statistical models 
describe associations; imbuing them with a causal or even interventionist interpretation requires strong additional assumptions (for guidance, see Elwert, 2013; Rohrer, 2018; Shmueli, 2010).

In fact, you can run into the same difficulties when you apply the interventionist mental shortcut in the absence of collinearity: In the dataset shown in Figure 4, it is impossible to change the second predictor without also changing the first since the first is a transformation of the second. Yet the two variables are not collinear, since the transformation is completely nonlinear. Or say you want to model quality ratings of texts in terms of the number of words in the text ("tokens"), the number of unique words in the text ("types"), and the type/token ratio. The model will output estimated coefficients for the three predictors, but as an analyst you should realise that it is impossible to find two texts differing in the number of tokens but having both the same number of types and the same type/token ratio: If you change the number of tokens and keep constant the number of types, the type/token ratio changes, too.

A final mental shortcut that is laid bare in the presence of collinearity is conflating a measured variable with the theoretical construct that this variable is assumed to capture. Conflating measurements and constructs can completely invalidate the conclusions drawn from a model even in the absence of collinearity (see Berthele and Vanhove, 2020; Brunner and Austin, 2009; Loftus, 1978; Wagenmakers et al., 2012; Westfall and Yarkoni, 2016). The literature on lexical diversity offers another case in point. The type/token ratio (TTR) discussed in the previous paragraph is one of several possible measures of a text's lexical diversity. If you take a collection of otherwise comparable texts, chances are that the longer texts tend to have lower TTR values (see Malvern et al., 2004, Chapter 2). This text-size dependence has led quantitative linguists to abandon the use of the TTR, even though the relationship in any given dataset need not be that strong (see Figure 7 for an example).

However, the reason why researchers have abandoned the use of the TTR is not collinearity per se. Rather, it is that the TTR is a poor measure of what it is supposed to capture, viz., the lexical diversity displayed in a text. Specifically, because of the statistical properties of language, the TTR is pretty much bound to conflate a text's lexical diversity with its length. The negative correlation between the TTR and text length is not a big problem for statistical modelling, but it is a symptom of a more fundamental problem: A measure of lexical diversity should not as a matter of fact be related to text length. The fact that the TTR is shows that it is a poor measure of lexical diversity. This problem

\section{$\begin{array}{llll}0.4 & 0.6 & 0.8 & 1.0\end{array}$}

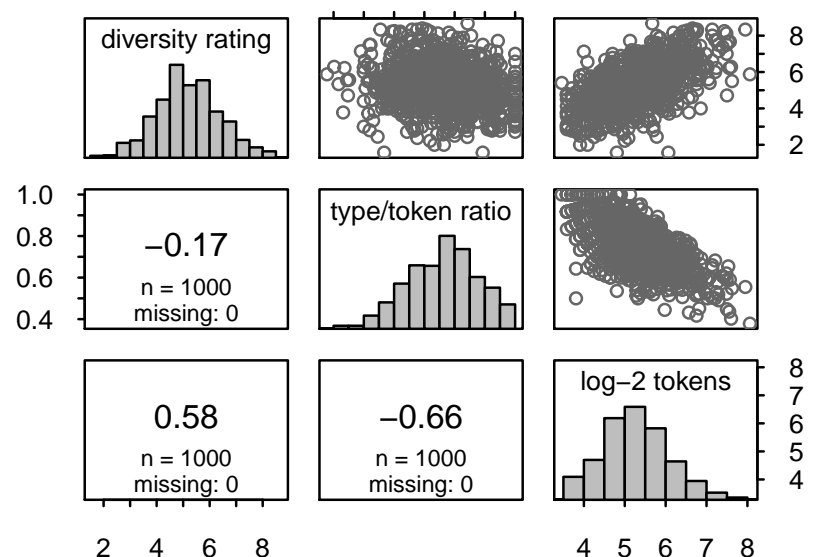

Figure 7 . The type/token ratio tends to be negatively correlated with text length (here: $\log -2$ number of tokens). But the problem is not that the type/token ratio is collinear with text length; it is that the type/token ratio also measures something it is not supposed to measure (length) and is a poor measure of what it is supposed to measure (lexical diversity, represented here by human ratings). Data from the French corpus published by Vanhove et al., 2019.

is hidden if researchers mentally equate the TTR with the construct of lexical diversity rather than remaining cognizant of the fact that it is but an attempt to quantify the construct-and not a successful one at that.

To be clear, it is not necessarily a problem that measures of lexical diversity empirically correlate with text length. After all, it is possible that the lexical diversity of longer texts is greater than that of shorter texts or vice versa: Texts may be pithy but lexically diverse if the writers often used le mot juste instead of elaborate circumlocutions, and long texts may be lexically more diverse than shorter ones if they were written by more sophisticated writers with more to tell. The problem with the TTR is that it almost necessarily correlates with text length, even if, at the construct level, the texts' lexical diversity does not. For instance, if you take increasingly longer snippets of texts from the same book, you will find that the TTR goes down (see Tweedie and Baayen, 1998). This does not mean that the writer's vocabulary skills went down in the process of writing the book, but that $\mathrm{s} /$ he had to reuse common words (e.g., articles, pronouns, prepositions, copula verbs, common or important content words). More generally, if your predictors correlate strongly when they are not supposed to, your problem is not collinearity, but it may be that in trying to capture one construct, you have also captured the one represented by the other predictor.

In sum, the interpretational challenges encountered 
when predictors are collinear are not caused by the collinearity itself but by mental shortcuts that may lead researchers astray even in the absence of collinearity.

\section{Collinearity does not require a statistical solution}

I have argued that collinearity is not a genuine statistical problem, so I do not think it should be addressed by statistical means. Let's take a closer look at some popular strategies that analysts resort to when their predictors are collinear and the repercussions of these strategies.

\section{Residualising predictors}

The first popular strategy for dealing with collinearity is to residualise one collinear predictor against the other. This means that one of the predictors is fitted as the dependent variable in a regression model with the other predictor(s) as the independent variable(s). The estimated residuals are extracted from this model and then used as a replacement for the original predictor in the multiple regression model. York (2012) and Wurm and Fisicaro (2014) comprehensively discuss the consequences of this approach; Figure 8 highlights the main points.

As seen in the top left and bottom right panels, residualising one of the predictors against the other and using these residuals in lieu of the original predictor does not bias the estimates for the residualised predictor relative to the original true parameter values in Equation (1). It also does not reduce the sample-to-sample variability of these estimates. So, as far as the residualised predictor is concerned, there is no downside or upside to this approach. However, as seen in the top right and bottom left panels, the estimates of the residualiser (i.e., the predictor that was not residualised) show less sample-to-sample variability, but they are substantially biased relative to the original true parameter values in Equation (1) when the original predictors are collinear. ${ }^{2}$ The reason for this is that any variance in the outcome that could be accounted for by both predictors is now assigned wholly to the residualiser (see York, 2012).

Residualising one of the predictors against the other, then, changes the meaning of the estimated coefficient for the residualiser in a way that I suspect is opaque to most analysts and consumers. In fact, I cannot wrap my head around the sentence that I am about to foist upon you: What, according to the model, would be the mean difference if you took a large group of data points that differed by one unit in the residualiser but whose other predictor values differed by the same amount and in the same direction from the values that you would expect this predictor to have based on the linear association between it and the residualiser in the sample?
(Everything following but describes what it means for the estimated residuals to be held constant.) Perhaps such estimates can be useful, but hardly more than once in a blue moon.

\section{Dropping collinear predictors}

A second approach is to drop one or more of the collinear predictors from the model. I have no problem with this approach per se. But the problem that it solves is not collinearity but rather that the original model was misspecified. This approach only represents a solution if the new model is capable of answering the research question since, crucially, estimated coefficients from models with different predictors do not have the same meaning.

For instance, say you are interested in the association between L2 grammatical knowledge and L2 reading proficiency and you fit a model with L2 reading test scores as the outcome, the learners' scores on an L2 grammar test as the focal predictor and their scores on an L2 vocabulary test as a 'control variable.' If you decide to drop the vocabulary test scores from the model because of their correlation with the grammar test scores, you change the meaning of the estimate of the coefficient for the grammar test scores. In the full model, this estimate captures the mean difference in reading proficiency between learners with the same vocabulary score but with a one-unit difference in grammar test scores. In the reduced model, the estimate captures the mean difference in reading proficiency between learners with a one-unit difference in grammar test scores, regardless of their performance on the vocabulary test score. Either estimate may be useful for addressing the research question, but this depends on the research question, not on the degree of collinearity. If the reduced model makes more sense than the full model in the presence of collinearity, it would have also made more sense in the absence of collinearity.

Something to be particularly aware of is that by dropping one of the collinear predictors, you bias the estimates of the other predictors relative to their original parameter values as shown in Figure 9 (and see Note 2). The reason is that, thanks to their correlation with the dropped predictor, the remaining predictors can now do some of its job in accounting for variance in the outcome.

${ }^{2}$ I say "relative to the original true parameter values" since, technically, the estimates in the new model are not biased either, but they estimate something different from the estimates in the original model. 


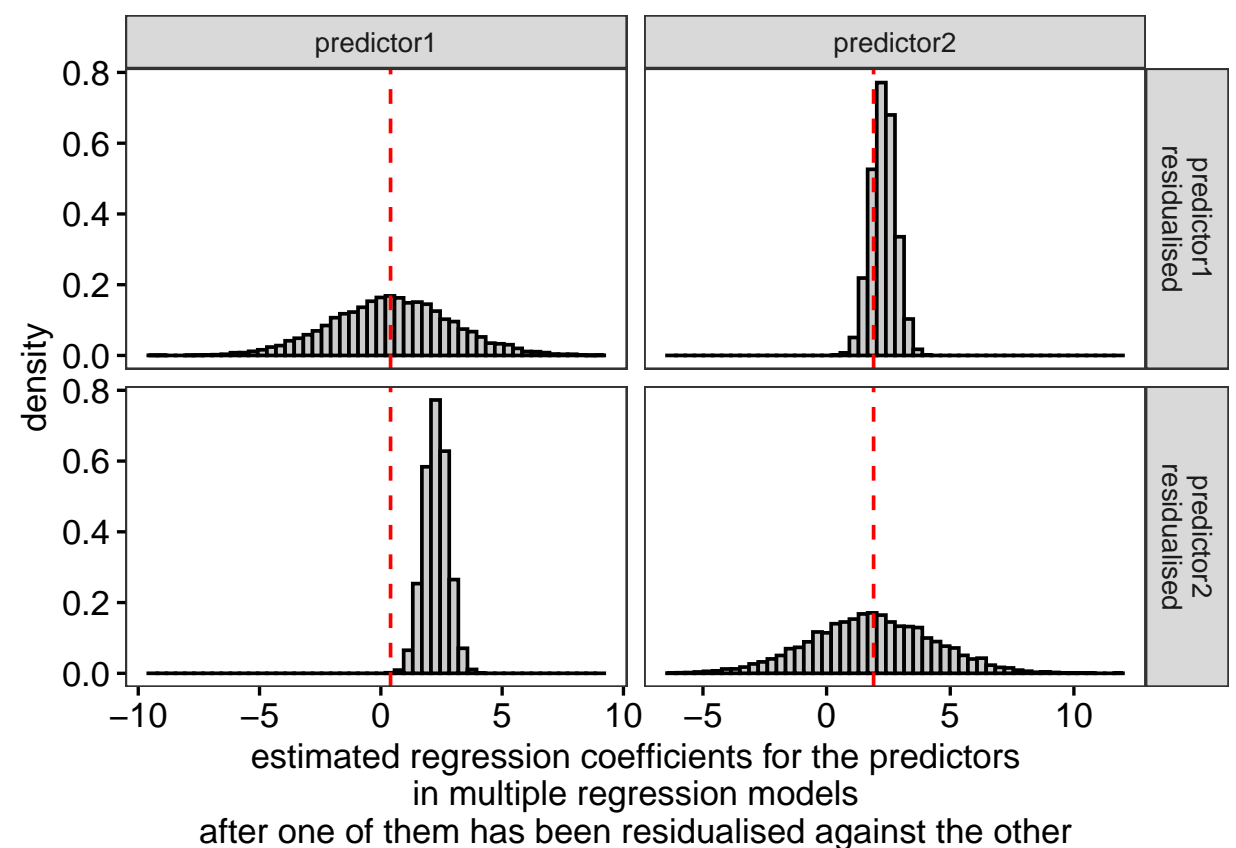

Figure 8. In the presence of strong collinearity $(r=0.98)$, residualising one of the predictors against the other does not bias the estimates for the residualised predictor or reduce their sample-to-sample variability (top left and bottom right), but it does bias the estimates of the residualiser (top right and bottom left) relative to the original parameter values (shown as dashed vertical lines).

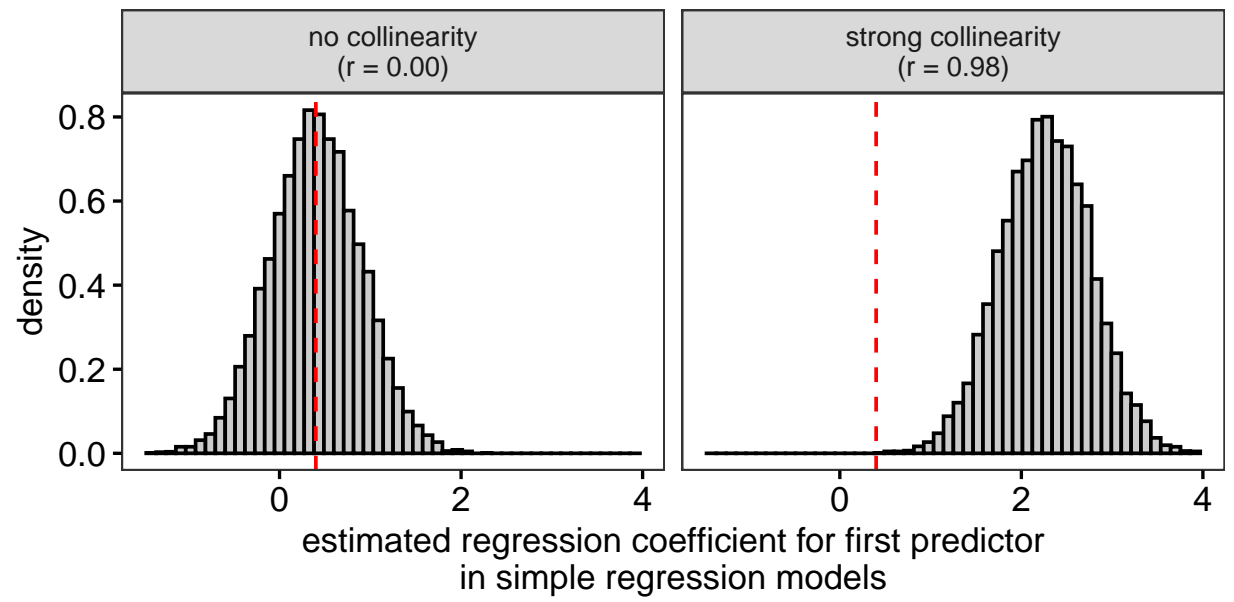

Figure 9. Dropping a collinear predictor changes the meaning of the estimate for the predictor retained (right panel). The reduced model now yields biased estimates of the original parameters (represented by the dashed vertical lines). When the predictors are perfectly orthogonal, this does not happen, but this is a special case.

\section{Averaging predictors}

A third strategy for dealing with collinearity is to compress the information in the collinear predictors into a smaller set of less strongly correlated predictors. For instance, analysts sometimes take the average of several (possibly $z$-standardised) predictors and use this average instead of the original predictors. Alternatively, they might submit these predictors to a principal component or factor analysis and extract one or more com- ponents or factors from this analysis to use these in lieu of the original predictors.

I do not mind this approach per se, either, but analysts should be aware that the meaning of their model estimates is now different from those in the model that they originally fitted. The estimates now express the model's best guess of the mean difference in the outcome when sampling a large number of data points that differ in one unit in the newly created variable but have 


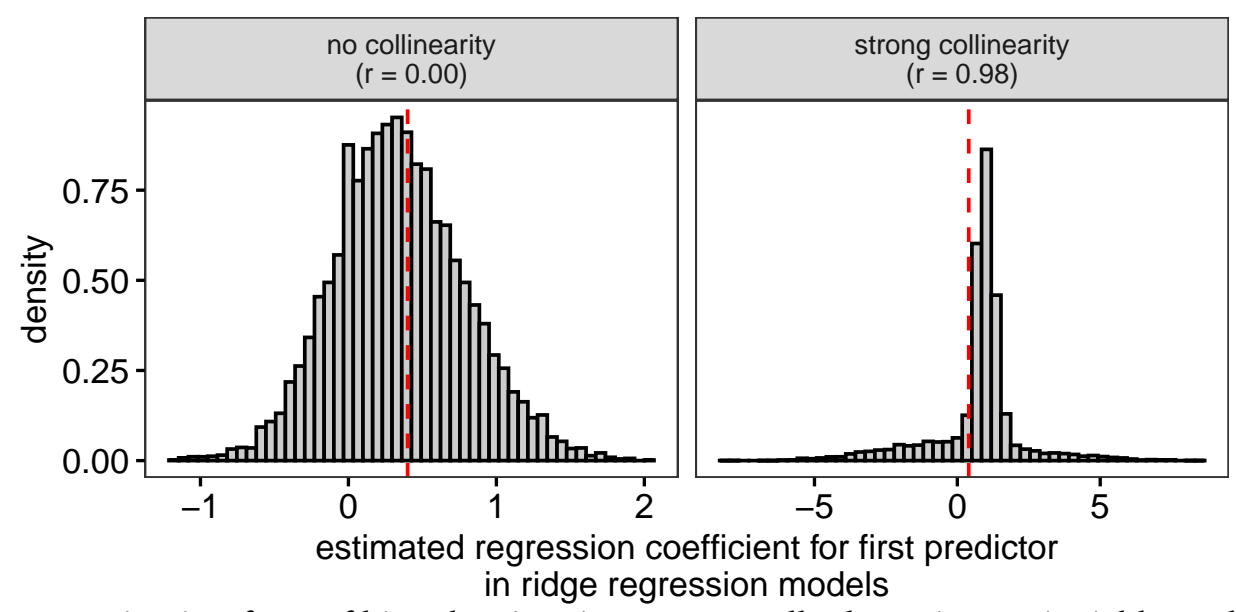

Figure 10. Ridge regression is a form of biased estimation, so naturally the estimates it yields are biased. When the predictors are orthogonal, all estimates are biased towards zero (left panel). When the predictors are collinear, the estimates for the weaker predictor are biased away from zero (right panel), whereas the estimates for the stronger predictor are biased towards zero (not shown).

otherwise identical predictor variables. Depending on the research question, such a model may be more defensible than the model originally fitted. But this depends on the research question, not on the degree of collinearity between the predictors.

\section{Using estimation methods such as ridge regression}

With independently and identically distributed errors (i.e., when the independence and homoskedasticity assumptions are met), ordinary least squares regression is guaranteed to yield unbiased estimates with the lowest possible sample-to-sample variability. Ridge regression and its cousins (lasso, elastic net) sacrifice unbiasedness in order to obtain estimates with an even lower sample-to-sample variability. This can be particularly useful in models optimised for predicting (as opposed to describing or explaining; see Kuhn and Johnson, 2013; Shmueli, 2010). Since collinearity is associated with more variable estimates, it is understandable that ridge regression and the like are used to tackle it. But the result of using models that deliberately bias the estimates is, quite naturally, that you end up with biased estimates.

I illustrate this in Figure 10, for which I reanalysed the data underlying Figure 5 using ridge regression. (Details of the choice of the $\lambda$ parameter are available in the supplementary materials, but they are not important here.) For orthogonal predictors, all estimates are biased towards zero. For strongly collinear predictors, the estimates for the weaker predictor will be biased away from zero (shown in the figure), and those for the stronger predictor will be biased towards zero.

Biased estimation, then, reduces sampling variability in the estimates, but at the cost of, well, biased estimation. Moreover, the usefulness of standard errors and confidence intervals for ridge regressions and its cousins is contested (see Goeman et al., 2018), so a further drawback is debatable statistical inference. ${ }^{3}$

In sum, popular strategies to address collinearity involve giving up the unbiased estimates of ordinary least squares regression, redefining the statistical model so that it answers different questions from the original model, or both. As York (2012) writes,

\begin{abstract}
'Statistical 'solutions,' such as residualization that are often used to address collinearity problems do not, in fact, address the fundamental issue, a limited quantity of information, but rather serve to obfuscate it. It is perhaps obvious to point out, but nonetheless important in light of the widespread confusion on the matter, that no statistical procedure can actually produce more information than exists in the data." [p. 1384]
\end{abstract}

\section{Summary}

Collinearity is a form of lack of information that is already appropriately reflected in the output of your statistical model. When collinearity is associated with

\footnotetext{
${ }^{3}$ With informative prior distributions on the parameters, Bayesian models can yield fairly narrow posterior distributions (i.e., a fairly low degree of uncertainty) for the estimates even in the presence of collinearity. But this is achieved by virtue of incorporating information from outside the sample into the model by means of the prior distributions, not by conjuring information out of thin air.
} 
interpretational difficulties, these difficulties are not caused by the collinearity itself. Rather, they reveal that the model was poorly specified (in that it answers a question different from the one of interest), that the analyst has overly focused on significance rather than estimates and the uncertainty about them, or that the analyst took a mental shortcut in interpreting the model that could have also led them astray in the absence of collinearity. These shortcuts include failing to interpret parameter estimates conditional on all the other predictors in the model, lending a causal or interventionist interpretation to what is a descriptive model without proper justification, and conflating a measure with the construct that it is supposed to represent. Lastly, if you do decide to deal with collinearity, make sure you can still answer the question of interest and that any bias in the estimates can be justified.

\section{Author Contact}

Jan Vanhove, University of Fribourg, Department of Multilingualism, Rue de Rome 1, 1700 Fribourg, Switzerland. ORCID ID: https://orcid.org/ 0000-0002-4607-4836. Website: https://janhove. github.io.

I thank Twitter user @facupalacio12 for the reference to Morrissey and Ruxton (2018), and Johan Ferreira, Nick Brown, and Rickard Carlsson for their comments.

\section{Conflict of Interest and Funding}

The author declares no conflict of interest and did receive specific funding for the present work.

\section{Author Contributions}

$\mathrm{JV}$ was the sole author of this article. This article is based on a blog post with the same title (https:// janhove.github.io/analysis/2019/09/11/collinearity).

\section{Open Science Practices}

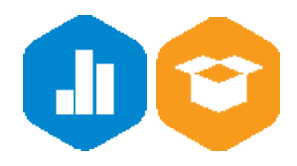

This article earned the Open Data and the Open Materials badge for making the data and materials openly available. It has been verified that the analysis reproduced the results presented in the article. The entire editorial process, including the open reviews, are published in the online supplement.

\section{References}

Berthele, R., \& Vanhove, J. (2020). What would disprove interdependence? Lessons learned from a study on biliteracy in Portuguese heritage language speakers in Switzerland. International Journal of Bilingual Education and Bilingualism, 23(5), 550-566. https :// doi.org / 10.1080/ 13670050.2017 .1385590

Brunner, J., \& Austin, P. C. (2009). Inflation of Type I error rate in multiple regression when independent variables are measured with error. Canadian Journal of Statistics, 37(1), 33-46. https: //doi.org/10.1002/cjs.10004

Elwert, F. (2013). Graphical causal models (S. L. Morgan, Ed.). In S. L. Morgan (Ed.), Handbook of causal analysis for social research. Dordrecht, The Netherlands, Springer. https:// doi.org/ 10.1007/978-94-007-6094-3\_13

Goeman, J., Meijer, R., \& Chaturvedi, N. (2018). L1 and L2 penalized regression models. https:// cran.r-project.org/web/packages/penalized/ vignettes/penalized.pdf

Kuhn, M., \& Johnson, K. (2013). Applied predictive modeling. New York, Springer. https://doi.org/10. 1007/978-1-4614-6849-3

Loftus, G. R. (1978). On interpretations of interactions. Memory \& Cognition, 6(3), 312-319.

Malvern, D., Richards, B., Chipere, N., \& Durán, P. (2004). Lexical diversity and language development: Quantification and assessment. Basingstoke, UK, Palgrave Macmillan. https://doi. org/10.1007/978-0-230-51180-4

McElreath, R. (2020). Statistical rethinking: A Bayesian course with examples in $R$ and Stan (2nd). Boca Raton, FL, CRC Press.

Miller, G. A., \& Chapman, J. P. (2001). Misunderstanding analysis of variance. Journal of Abnormal Psychology, 110(1), 40-48. https : / / doi.org / 10.1037/0021-843X.110.1.40

Morrissey, M. B., \& Ruxton, G. D. (2018). Multiple regression is not multiple regressions: The meaning of multiple regression and the non-problem of collinearity. Philosophy, Theory, and Practice in Biology, 10(3). https:// doi.org/10.3998/ ptpbio.16039257.0010.003

O'Brien, R. M. (2007). A caution regarding rules of thumb of variance inflation factors. Quality \& Quantity, 41, 673-690. https:// doi.org/10. 1007/s11135-006-9018-6

Rohrer, J. M. (2018). Thinking clearly about correlations and causation: Graphical causal models for observational data. Advances in Methods and 
Practices in Psychological Science, 1(1), 27-42. https://doi.org/10.1177/2515245917745629

Schmidt, F. L. (1996). Statistical significance testing and cumulative knowledge in psychology: Implications for training of researchers. Psychological Methods, 1, 115-129. https://doi.org/10.1037/ 1082-989X.1.2.115

Shmueli, G. (2010). To explain or to predict? Statistical Science, 25(3), 289-310. https://doi.org/10. 1214/10-STS330

Tweedie, F. J., \& Baayen, R. H. (1998). How variable may a constant be? Measures of lexical richness in perspective. Computers and the Humanities, 32(5), 323-352. https://doi.org/10.1023/A: 1001749303137

Vanhove, J., Bonvin, A., Lambelet, A., \& Berthele, R. (2019). Predicting perceptions of the lexical richness of short French, German, and Portuguese texts using text-based indices. Journal of Writing Research, 10(3), 499-525. https:// doi.org/10.17239/jowr-2019.10.03.04
Wagenmakers, E.-J., Krypotos, A.-M., Criss, A. H., \& Iverson, G. (2012). On the interpretation of removable interactions: A survey of the field 33 years after Loftus. Memory \& Cognition, 40(2), 145-160. https://doi.org/10.3758/s13421011-0158-0

Westfall, J., \& Yarkoni, T. (2016). Statistically controlling for confounding constructs is harder than you think. PLOS ONE, 11(3), e0152719. https: //doi.org/10.1371/journal.pone.0152719

Wurm, L. H., \& Fisicaro, S. A. (2014). What residualizing predictors in regression analyses does (and what it does not do). Journal of Memory and Language, 72, 37-48. https://doi.org/10.1016/ j.jml.2013.12.003

York, R. (2012). Residualization is not the answer: Rethinking how to address multicollinearity. Social Science Research, 41, 1379-1386. https:// doi.org/10.1016/j.ssresearch.2012.05.014 